\title{
STUDIES OF FLIGHT TRAJECTORY OPTIMIZATION AND THE WORKLOADS OF AIR TRAFFIC CONTROL OFFICERS
}

\author{
Weiyang Sun \\ School of Mechanical \& Aerospace Engineering, Nanyang Technological University, 50 \\ Nanyang Avenue, Singapore 639798, Singapore
}

Wei-Chuen Wallace Ong

School of Mechanical \& Aerospace Engineering, Nanyang Technological University, 50 Nanyang Avenue, Singapore 639798, Singapore

Zhao-Wei Zhong (corresponding author)

School of Mechanical \& Aerospace Engineering, Nanyang Technological University, 50 Nanyang Avenue, Singapore 639798, Singapore

\begin{abstract}
We studied a methodology for flight trajectory optimization, and also the workloads of air traffic control officers (ATCOs). Case studies were conducted through simulations for flight trajectory optimization. The aircraft model was A320-200s, which is pre-dominantly utilized in Southeast Asia for short to medium range flights. Fuel savings were computed for selected routes, and were compared with that of existing operations and flights simulations, which revealed significant fuel savings. The research also determined the coefficients of ATCOs' workloads and demonstrated dynamic sectorization in selected airspace of Southeast Asia. It was found that dynamic sectorization was more efficient than static sectorization in balancing the workloads of ATCOs, reducing the standard deviation by $50 \%$ and the balance of workloads among sectors by $12.9 \%$.
\end{abstract}

\section{KEYWORDS}

Balance of Workloads, Controller Workload, Dynamic Sectorization, Flight Trajectory Optimization, Fuel Savings

Weiyang Sun was a student at School of Mechanical and Aerospace Engineering, Nanyang Technological University, Singapore. Email: wsun002@e.ntu.edu.sg

Wei-Chuen Wallace Ong was a student at School of Mechanical and Aerospace Engineering, Nanyang Technological University, Singapore, Email: wallaceongwc@gmail.com

Zhao-Wei Zhong is a faculty member at School of Mechanical and Aerospace Engineering, Nanyang Technological University, Singapore. E-mail address: mzwzhong@ntu.edu.sg 


\section{INTRODUCTION}

Because of developing economies and emerging markets in Asia Pacific, compared to other regions, this region has faster air transport growths (Tee and Zhong, 2018). One study reported in this paper is an introductory step forward for Southeast Asia, due to the limited studies in air traffic management in this region until recent years (Zhong et al., 2017). Experimental constants and multipliers used in conventional algorithms governing trajectorybased optimization might not be possible for the computation of the modified algorithm in this paper. This was overcome, which yielded a good approximation, as shown by the results of the study presented in section 2. For selected routes in Southeast Asia, significant fuel savings could be computed compared with existing operations and flights simulations.

Air traffic control officers (ATCOs) ensure the safety of aircraft and smooth traffic flow, and one major factor that determines their workloads is the sectorization of airspace (Kopardekar et al., 2007). A larger sector results in increased ATCOs' workloads due to increased aircraft entry rates and time to monitor conflicts. ATCOs' routine workloads are also affected by the way the airspace sectors are partitioned (Trong et al., 2016). With an increasing demand for air travel in Southeast Asia (Phyoe et al., 2016; Raheja and Zhong, 2018), it is even more important to ensure that ATCOs are not overloaded (Majumdar and Ochieng, 2002). The growth in air traffic volumes has resulted in an increasing strain on air transport systems, and the airspace capacity is closely related to ATCOs' workloads (Zhong et al., 2016). Unexpected poor weather conditions such as heavy storms or ashes from volcano eruptions may lead to airport closures and changes in flight paths (Sheth et al., 2013; Xie and Zhong, 2016a; Lim and Zhong, 2018).

The current method to manage the increasing demand is to divide the airspace into more sectors, so that ATCO workloads can be manageable. However, small sectors result in more resource fragmentation. There is a size limit for a very small sector to be further subdivided (Foo and Zhong, 2017). One approach to address this issue is dynamically sectorizing the airspace and keeping the ATCO workloads manageable (Foo and Zhong, 2018).

With a decrease in workloads of ATCOs for monitoring and control, they can better handle strategic control problems and manage traffic under bad weather conditions (Amin et al., 2013; Xie and Zhong, 2016b). Thus, in the second part of this work, various values of the coefficients were used to study how they affected the imbalance of workloads and the standard deviation in static and dynamic sectorization. Lastly, as dynamic sectorization has not been implemented in Southeast Asia, this work also examined if the application of dynamic sectorization to 
selected airspace of Southeast Asia could better balance the workloads of ATCOs and lower the standard deviation throughout various times of the day, as detailed in section 3.

\section{STUDY OF FLIGHT TRAJECTORY OPTIMIZATION}

The foundation begins with the derivation of the atmospheric properties, which contributes to subsequent aerodynamic calculations and lateral optimization in the relevant altitude wind fields. The input of the weather conditions is automated from a weather model built. The only necessary user input would be the beginning flight altitude for the cruising phase. Subsequently through the application of an aircraft model constructed from Base of Aircraft Database (BADA), the aerodynamic properties are derived. The computation leads to an altitude optimization, which then is coupled with the lateral optimization in an iterative process until results converge. The number of the iterative process is based on the user's input of waypoints. At least 7 waypoints are used for convergence (Sun, 2016).

The cost function (Equation 1) proposed by $\mathrm{Ng}$ et al. (2012) is adopted.

$$
J=\int_{t_{i}}^{t_{f}}\left[C_{t}+C_{f} F(m, h, V)\right] d t
$$

$C_{t}$ represents the cost coefficient of time, one of the key considerations for airliners in terms of operational functionality. The cost coefficient of fuel, $C_{f}$, is the key consideration of this study. As the aircraft model under study is A320-200s, which has turbofan engines, the important relationship of altitude, Mach number and velocity of a turbofan is accounted for (Roth and Mavris, 2001; Turgut et al., 2009):

- $\quad$ Specific Fuel Consumption (SFC) is near constant with altitude.

- $\quad$ SFC increases with an increase in free stream velocity.

- $\quad$ SFC increases with an increase in Mach number.

The optimization form proposed by $\mathrm{Ng}$ et al., is the derivative of fuel consumed with respect to altitude changes resulting in Equation 2 ( $\mathrm{Ng}$ et al., 2012):

$$
\frac{d f}{d h}=\frac{C_{f c r}}{1000}\left(D \cdot \frac{d S F C}{d h}+S F C \cdot \frac{d D}{d h}\right)
$$


Referring to Equation 2, changes of SFC with altitude are set to be zero $\left(\frac{d S F C}{d h}=0\right)$, as per discussed using the turbofan's performance characteristic, and only the latter term is to be considered ( $\mathrm{Ng}$ et al., 2011; $\mathrm{Ng}$ et al., 2012).

A reduction in drag with respect to altitude would be the key premise for consideration in lateral optimization. Therefore, through cross-product, this key relationship can be obtained as shown in Equation 3 (Sun, 2016).

$$
\frac{d D}{d h}=\frac{d D}{d \rho} \cdot \frac{d \rho}{d h}
$$

$\frac{d \rho}{d h}$ is not zero, as density changes with altitude based on the ISA model adopted (which can be found in the later part of this section). Therefore, $\frac{d D}{d \rho}=0$ for $\frac{d D}{d h}=0$ ( $\mathrm{Ng}$ et al., 2011; $\mathrm{Ng}$ et al., 2012). This would result in the focus being on $\frac{d D}{d \rho}$, which produces Equation 4 (Sun, 2016):

$$
\frac{d D}{d \rho}=\frac{1}{2} \rho V^{2} S\left(C_{D o}+K C_{L}^{2}\right)
$$

A modification done was replacing the experimental constants used in $\mathrm{Ng}$ 's original equation, which were obtained through a pre-existing dataset. Replacing this limitation with an empirical formula, the Oswald's efficiency (referring to $K$ in Equation 4 ) for large commercial transport aircraft was calculated and found to be in the range of 0.83-0.85 (Nita and Scholz, 2012).

The range for consideration is only at steady level flight, in which cruising occurs $(n=1)$. This would result in the governing aerodynamic equations (Equations 5 to 8) (Sun, 2016).

$$
\begin{gathered}
L=W=m g \\
C_{L}=\frac{2 m g}{S V^{2} \rho}-C_{L o} \\
\frac{d D}{d \rho}=\frac{1}{2} \rho V^{2} S\left(C_{D o}+\frac{1}{\pi e A R} \cdot\left(\frac{2 m g}{S V^{2} \rho}-C_{L o}\right)^{2}\right) \\
\frac{d D}{d \rho}=\frac{1}{2}\left(V^{2} S C_{D o}+\frac{1}{\pi e A R} C_{L o}^{2} S V^{2}-\frac{1}{\pi e A R} \cdot \frac{4 m^{2} g^{2}}{S V^{2} \rho^{2}}\right)=0
\end{gathered}
$$

The papers from ( $\mathrm{Ng}$ et al., 2011; $\mathrm{Ng}$ et al., 2012) showed a relationship, which is expressed in Equation 9: 


$$
\frac{4 m^{2} g^{2} K}{C_{D o}} \cdot \frac{1}{S^{2} V^{4}}=\rho^{2}
$$

Another modification made by Sun (2016) alters Equation 9 into Equation 10:

$$
\frac{4 m^{2} g^{2} K}{C_{D o}+\frac{1}{e \pi A R} C_{L o}^{2}} \cdot \frac{1}{S^{2} V^{4}}=\rho^{2}
$$

Sun (2016) has added the additional constant that accounts for the zero lift coefficients. This is not prominent for symmetrical airfoils. However, as the exact nature of commercial airlines airfoils is unknown, it is more reasonable to assume a non-symmetrical general case.

A third modification was made by Sun (2016) on the initial condition provided by $\mathrm{Ng}$ et al. (refer to Equation 2). This modification was necessary because of the difference in atmospheric models adopted. Sun has adopted the ISA model presented by Cavcar (2000), and the ideal gas model has been applied to Equation 11 (Sun, 2016).

$$
\frac{d f}{d h}=\frac{C_{f c r}}{1000}\left(S F C \cdot \frac{d D}{d \rho} \cdot \frac{P}{R T}\right)=0
$$

$C_{f c r}, \mathrm{SFC}$ and $\frac{d \rho}{d h}$ are all not zero. Therefore, $\frac{d D}{d \rho}=0$ for $\frac{d f}{d h}=0$.

This produces the altitude optimization equation that was presented by $\mathrm{Ng}$ et al. The equation has been modified by Sun (2016) to include the lift coefficient at zero angle of attack, and thus is a generalized equation applicable to even non-symmetric airfoils.

It has been found through experimental results as presented by $\mathrm{Ng}$ et al. in Equation 12 , that fuel consumption is a logarithmic function of the square of density, which is co-related to the minimum drag to altitude ratio found in the above equations ( $\mathrm{Ng}$ et al., 2011; $\mathrm{Ng}$ et al., 2012).

$$
f=\ln \left(\rho^{2}\right)
$$

The fuel consumption model presented has been modified to Equation 13 (Sun, 2016) by substituting Equation 10 into Equation 12.

$$
f=\ln \left(\frac{4 m^{2} g^{2} K}{C_{D o}+\frac{1}{e \pi A R} C_{L o}^{2}} \cdot \frac{1}{S^{2} V^{4}}\right)
$$

Equation 13 is a generalized equation, which can be used for any airfoil available, symmetrical or non-symmetrical. Therefore, the optimum altitude is found using Equation 14 or 15. 
At or below Tropopause $(11,000 \mathrm{~m})(\mathrm{Ng}$ et al., 2012):

$$
h_{\text {optimum }}=\left(1-e^{\frac{-f K_{T} R}{2\left(g+K_{T} R\right) \rho_{o}^{2}}}\right) \cdot\left(\frac{1000 T_{o}}{6.5}\right)
$$

Where $\mathrm{K}_{\mathrm{T}}$ refers to the temperature gradient associated with that at or below Tropopause.

Above Tropopause (11,000 m) (Ng et al., 2012):

$$
h_{\text {optimum }}=\frac{-f R T_{\text {trop }}}{2 g \rho_{\text {trop }}^{2}}+11000
$$

A flight simulator group from Singapore Virtual Airlines Group and Singapore vACC helped to conduct the simulations (Sun, 2016). Different pilots piloted the flights from Singapore to Cambodia, to ensure that regardless of the human element involved, the System Optimization could still provide a flight route to achieve minimum fuel consumed. The simulations used Microsoft Flight Simulator X, with weather condition inputs taken from Active Sky Next for FSX. Three flights flew under actual current flight routes being flown by airline operators (termed Control). Three flights flew using trajectory solutions with the modified method (termed Model 1) (Sun, 2016). Three flights flew using trajectory solutions with the original method (termed Model 2) ( $\mathrm{Ng}$ et al., 2012). The aircraft model used was an A320-200 at the maximum takeoff weight with a 180-pax capacity.

The trajectory solution produced by Model 1 represents a closer approximation to the altitude flown in the simulation studies, resulting in a better prediction in fuel savings. A higher altitude results in a lower atmospheric density, which reduces drag and thus indirectly contributes to better fuel savings. The variation of the simulated flight altitude is a natural occurrence due to wind gust experienced.

Control flight 1, experiment flight 1 (Model 1) and experiment flight 1 (Model 2) correspond to the same pilot; the only deviation is in terms of route taken. This is the same for flight sets 2 and 3. Maximum take-off weight was set at 77,000 kg. The fuel to be uploaded in respect to the payload accommodated was not specified, providing the pilots with the freedom to decide the amount of fuel to be uploaded. This was done so as to mimic real-life situations, providing the pilots with the flexibility of fuel-onboard (FOB). As it was conducted only on A320s, the variation in fuel-onboard only affected the range of the aircraft and not the overall fuel efficiency (Airbus, 2005). As such, the only requirement imposed for the decision of FOB for the simulator pilots was being sufficient to arrive at the destination and maintain an emergency reserve for any holding if applicable. 
In all three cases shown in Figure 1, Model 1 trajectory solution resulted in the least fuel consumed. It yielded $300-1020 \mathrm{~kg}$ fuel savings per flight. Flight route trajectories created by Model 2 yielded $280-930 \mathrm{~kg}$ fuel savings.

Figure 1: Simulated fule consumed

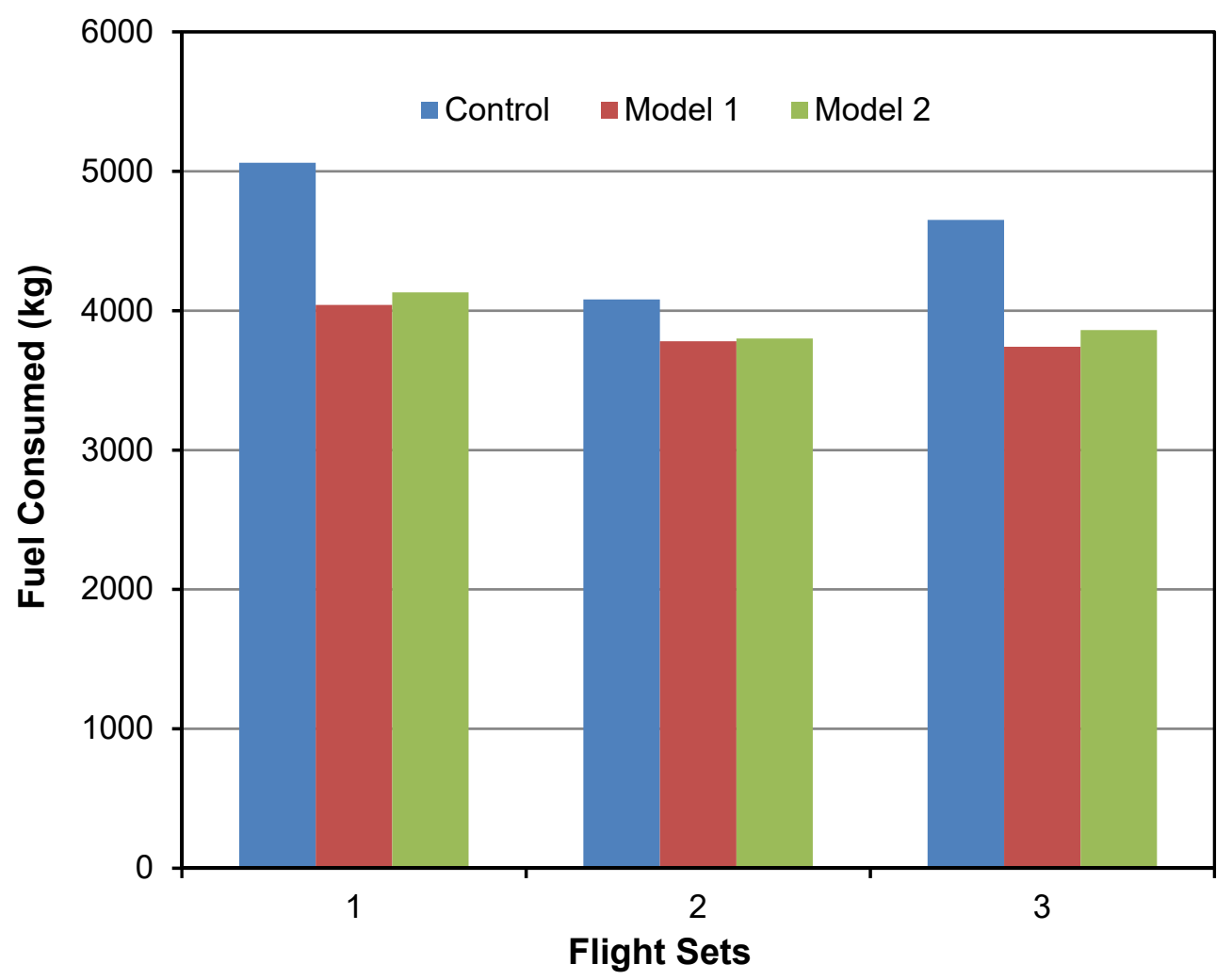

In Figure 2, the predicted fuel savings calculated with the algorithms are compared with the simulated flight fuel savings. Model 1, compared with the simulated flights, yields a better prediction. The improvement reduces the gap between the predicted and simulated results. 
Figure 2: Predicted fuel savings compared with the simulated flight fuel savings

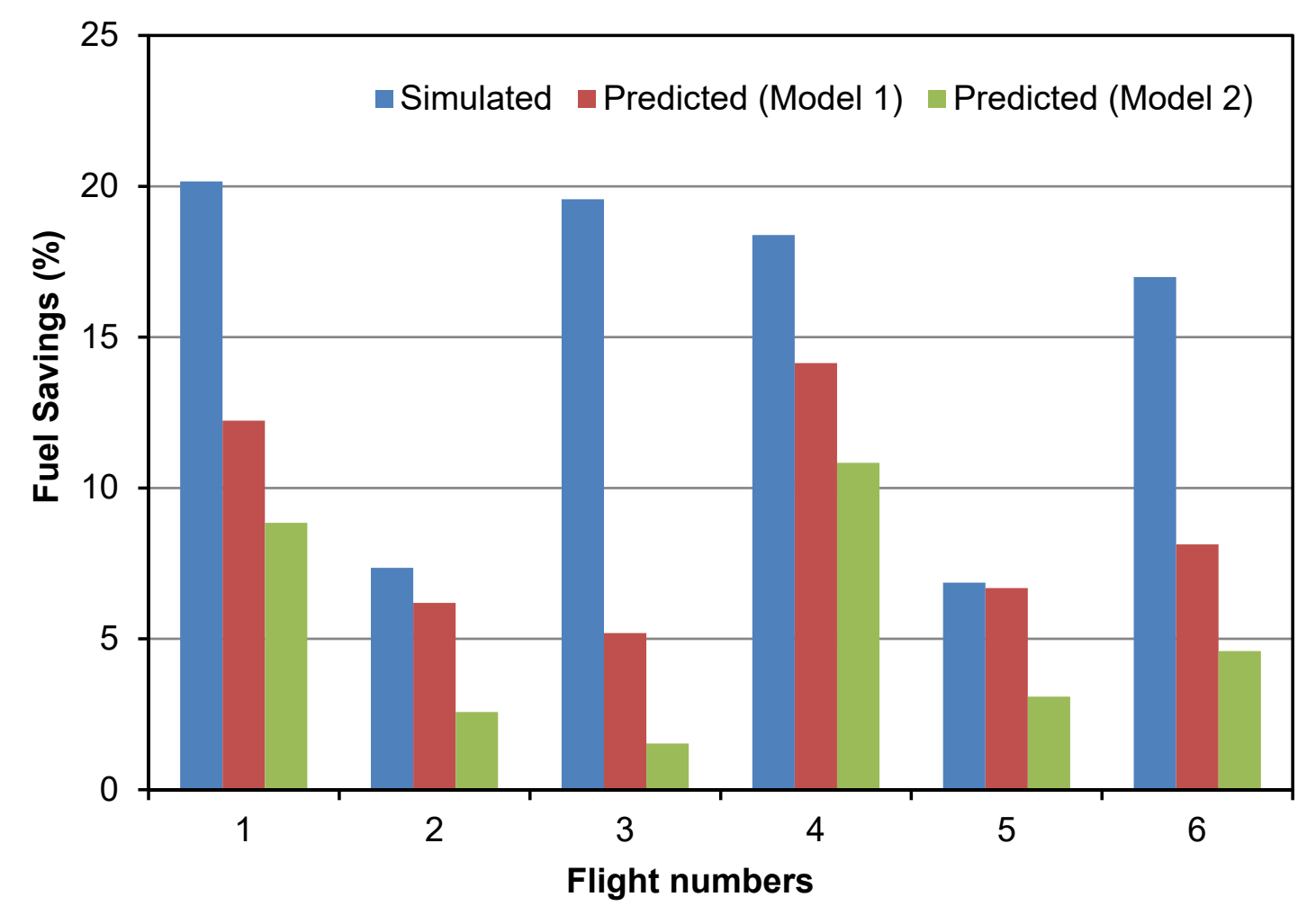

The differences between predicted and simulated fuel savings are shown in Figure 3. Model 1 provides a better prediction, and the differences in all 6 flights are $0.18-8.85 \%$. In the case of Model 2, the differences are 3.78-18.04\%. The modifications done for Model 1 can reduce the difference between simulated and predicted results. The ability of the algorithm to predict fuel consumed would help pilots utilizing a program to determine how much fuel to upload, reducing excess weight from excess fuel uploaded. A reduction in weight would lead to greater fuel savings in return.

As flights from Singapore to Cambodia occur on a daily basis, the fuel savings multiplied over a time period can result in a significant amount. Based on ICAO Carbon Emission methodology, an aircraft with a passenger capacity of 180 , flying from Singapore to Cambodia, produces $21207.6 \mathrm{~kg}$ of carbon emission per flight (International Civil Aviation Organization, 2014; Sun, 2016). A reduction in fuel consumed over the course of a year would lead to a significant amount of reduction in carbon emission. 
Figure 3: Differences between predicted and simulated fuel savings

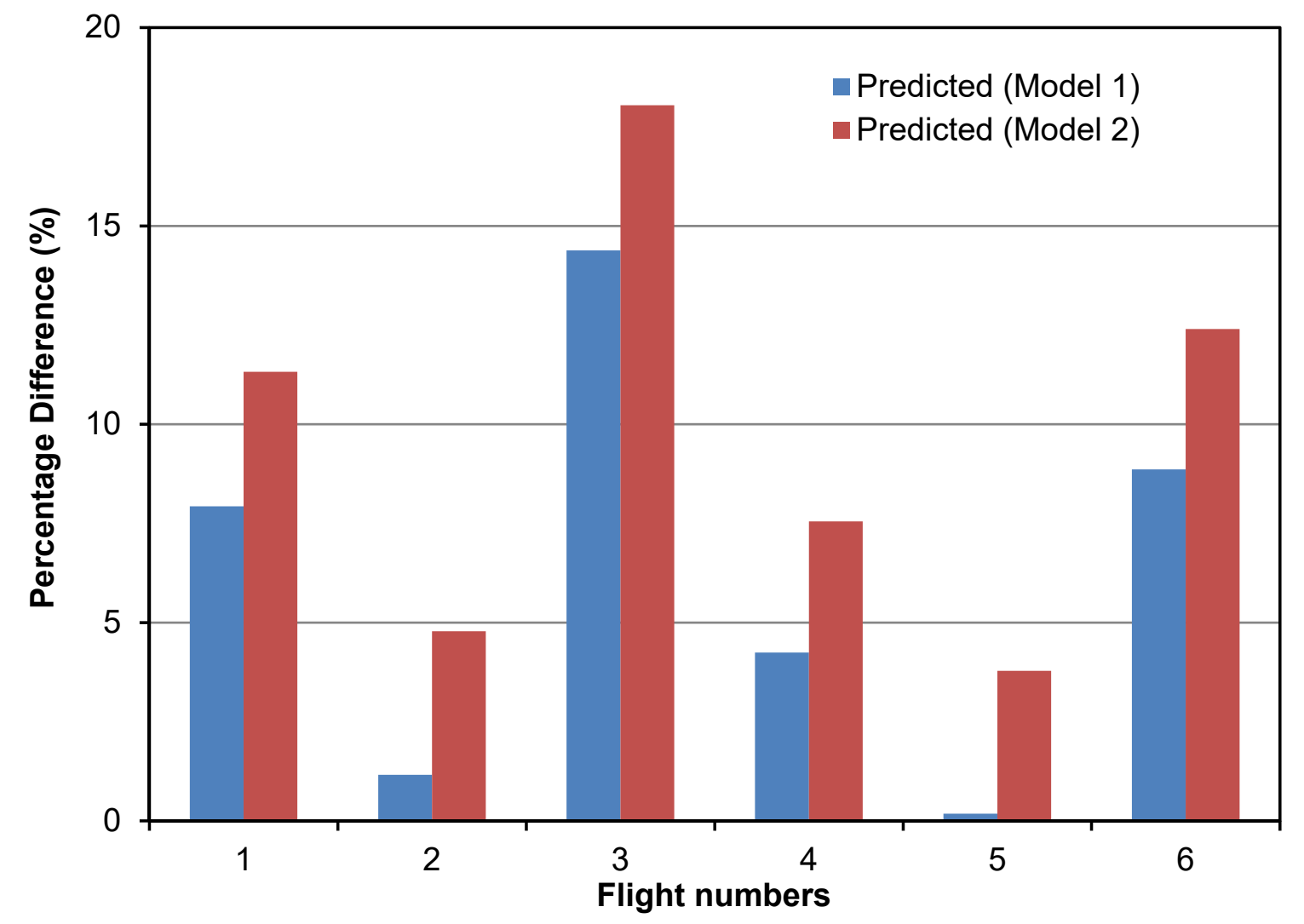

\section{STUDY OF ATCO WORKLOADS}

In this study, the state of equipment is treated as a constant, as the study is conducted with the comparison in the same country's airspace and the states of equipment in the various control centers are assumed to be similar (Ong, 2016). The workload experienced by ATCOs would only be affected by the situation in the airspace.

Therefore, the total workload experienced by ATCOs would be the combination of the following three components (Wang et al., 2010): (1) Monitoring workload, $W L_{\text {monitoring }}$ - This is the workload for monitoring flights in a sector and checking the trajectories of the aircraft in the sector. Monitoring workload depends on the air traffic and is proportionate to the number of flights within the sector throughout certain duration. (2) Coordination workload, $W L_{\text {coordination }}$ - This is the workload for coordinating during takeover or handover of flights to or from adjacent sectors. This can be information exchange between two controllers of adjacent sectors or between the controller and a pilot. (3) Conflict resolution workload, $W L_{\text {conflict }}$ - This is the workload to monitor two or more aircraft crossing a waypoint in opposite directions. This workload is proportional to the number of crossing aircraft. 
The total workload of an ATCO is therefore (Li et al., 2009):

$$
\begin{gathered}
W L_{\text {total }}=\alpha W L_{\text {monitoring }}+\beta W L_{\text {coordination }}+\gamma W L_{\text {conflict }} \\
\alpha \geq 0, \beta \geq 0, \gamma \geq 0 .
\end{gathered}
$$

Where $\alpha$ is the monitoring coefficient, $\beta$ is the coordination coefficient, and $y$ is the conflict resolution coefficient. The coefficients $\alpha, \beta$ and $\gamma$ are from the empirical study of air traffic workloads. However, because of the lack of data from relevant authorities, we assume the monitoring coefficient $\alpha$ to be 0.1 . $\beta$ and $\gamma$ are plotted against the imbalance to find the optimal value where the change of imbalance is less than $0.5 \%$. In this article, the unit of $W L_{\text {total }}$ would be based on the number of flights within the sector. Upon calculation of $W L_{\text {total }}$, the weight matrix $W$ is then created as follows (Von Luxburg, 2007).

$$
W=\left(w_{i j}\right)_{i, j=1, \ldots, n} .
$$

If $w_{i j}=0$, there are no flights over the flight route, and this means $a_{i j}=0$.

After the sectorization of the airspace, we determine the performance of the sectorization by comparing two properties, the coefficient of sector workload balancing and the standard deviation. To determine if the workloads of the various sectors are balanced, we compare the imbalance of workloads among the sectors, $c_{b a l}$, which is defined as (Li et al., 2010)

$$
c_{b a l}=\frac{W L_{\max }-W L_{\min }}{W L_{\max }} \times 100 \%
$$

Where $W L_{\text {max }}$ is the maximum sector workload and $W L_{\min }$ is the minimum sector workload. Hence, $c_{b a l}$ is the maximum difference among the sector workloads. The smaller this value is, the more balanced the sector workloads are.

The standard deviation percentage, $\sigma$, is defined as (Savai et al., 2010)

$$
\sigma=\frac{\sqrt{\frac{1}{N} \sum_{i=1}^{N}\left(W L_{i}-\mu\right)^{2}}}{\text { Average Workload }} \times 100 \%
$$

Where $\mu$ is the mean of the workloads of all the sectors. The standard deviation percentage, $\sigma$ measures how the workloads of the sectors deviate from the mean workload. The smaller this value is, the more balanced the sector workloads are.

The demand for air travel in Vietnam has been increasing in recent years. ATCOs in Hanoi FIR have to deal with an average of 30 to 35 flights per hour (Thanh Nien News, 2016). In this 
section, we do not consider military airports and flights, due to the lack of such flight information. Hanoi FIR is located north of Ho Chi Minh FIR and has three active airports, two international airports with larger traffic and one domestic airport. To ensure that proper measures can be taken to sectorize the airspace efficiently, it is important to get the optimal coefficients. In 2015, Hanoi FIR was sectorized into three sectors (Vietnam Aeronautical Information Centre, 2015).

The numbers of flights across various air routes were collected from FlightStats and matched to the numbering system of the nodes. Some of the air routes have zero flight and are not recorded (FlightStats, 2016).

Using the numbers of flights in Hanoi FIR for the whole day and the actual sectorization, we could find the imbalance of sectors, $c_{b a l}$ with the various values of $\beta$ and $\gamma$. The optimal value, where the change of imbalance was less than $0.5 \%$, was then noted (Ong, 2016). The numbers of flights were then used to analyze how the imbalance of workloads among the sectors and standard deviation changed, as the coefficient of coordination, $\beta$ and coefficient of conflict resolution, $\gamma$ changed. Figure 4 shows how the dynamic sectorization (Ong, 2016) of Hanoi FIR changes throughout the day based on the optimal coefficients achieved.

The imbalance of the sector workload and the standard deviations for the dynamic and actual sectorization with the optimal coefficients and other coefficients are then plotted in Figs. 5 and 6 respectively. The average values of $c_{b a l}$ and $\sigma$ are tabulated in Table 1 .

In Figs. 5 and 6, the red lines represent the imbalance of workload among the sectors and the standard deviations with the coefficients of $\beta=0.31$ and $\gamma=0.55$. The green lines are drawn with the coefficients of $\beta=0.1$ and $\gamma=0.1$ and the blue lines are drawn with the coefficients of $\beta=2$ and $\gamma=2$. The thicker lines represent the readings from the dynamic sectorization, while the thinner lines represent the readings from the actual sectorization (in 2015) of Hanoi FIR. 
Figure 4: Dynamic sectorization of Hanoi FIR
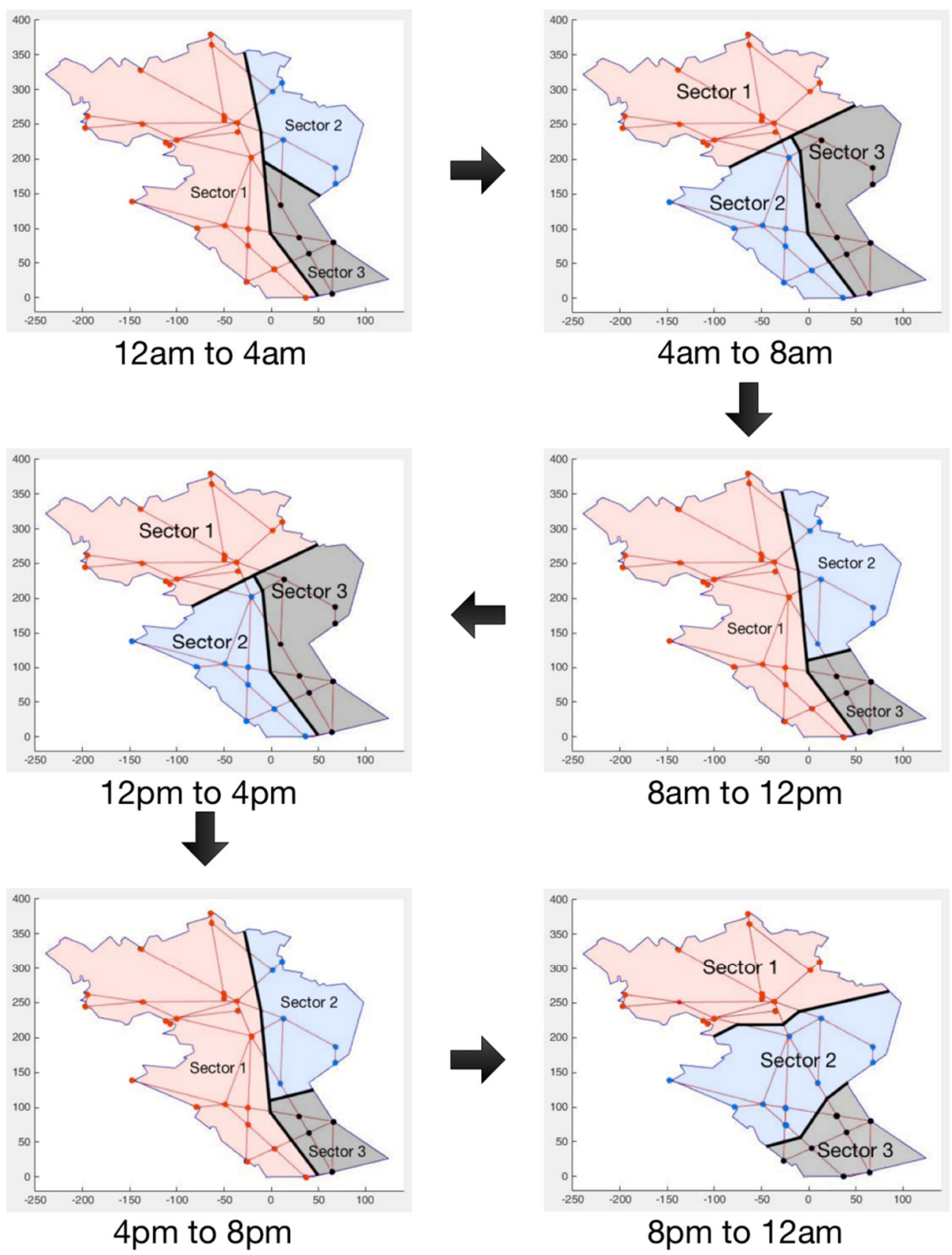
Figure 5: Graph of $\boldsymbol{c}_{\boldsymbol{b a l}}$ for both dynamic and actual sectorization

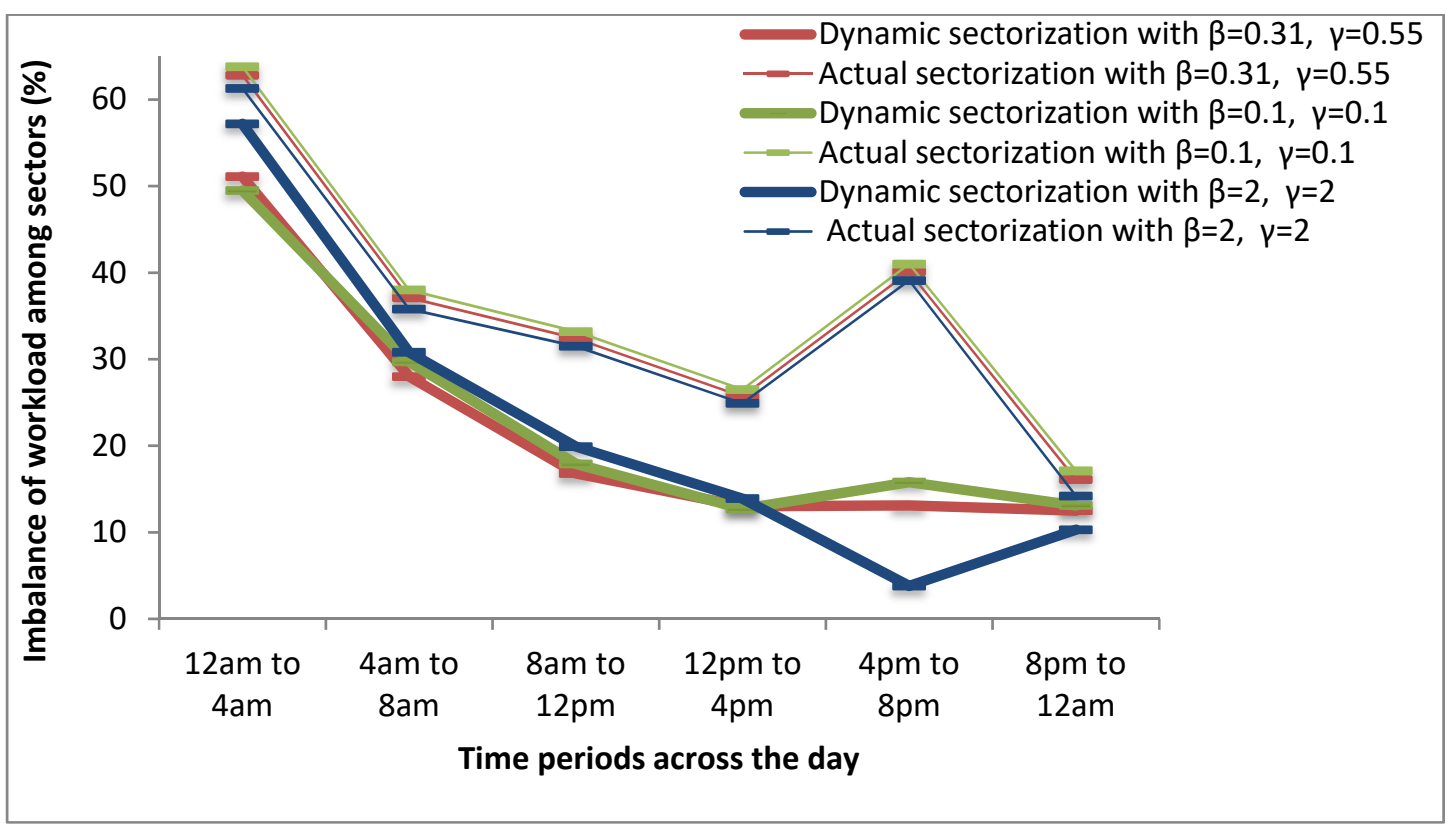

Figure 6: Graph of $\boldsymbol{\sigma}$ for both dynamic and actual sectorization

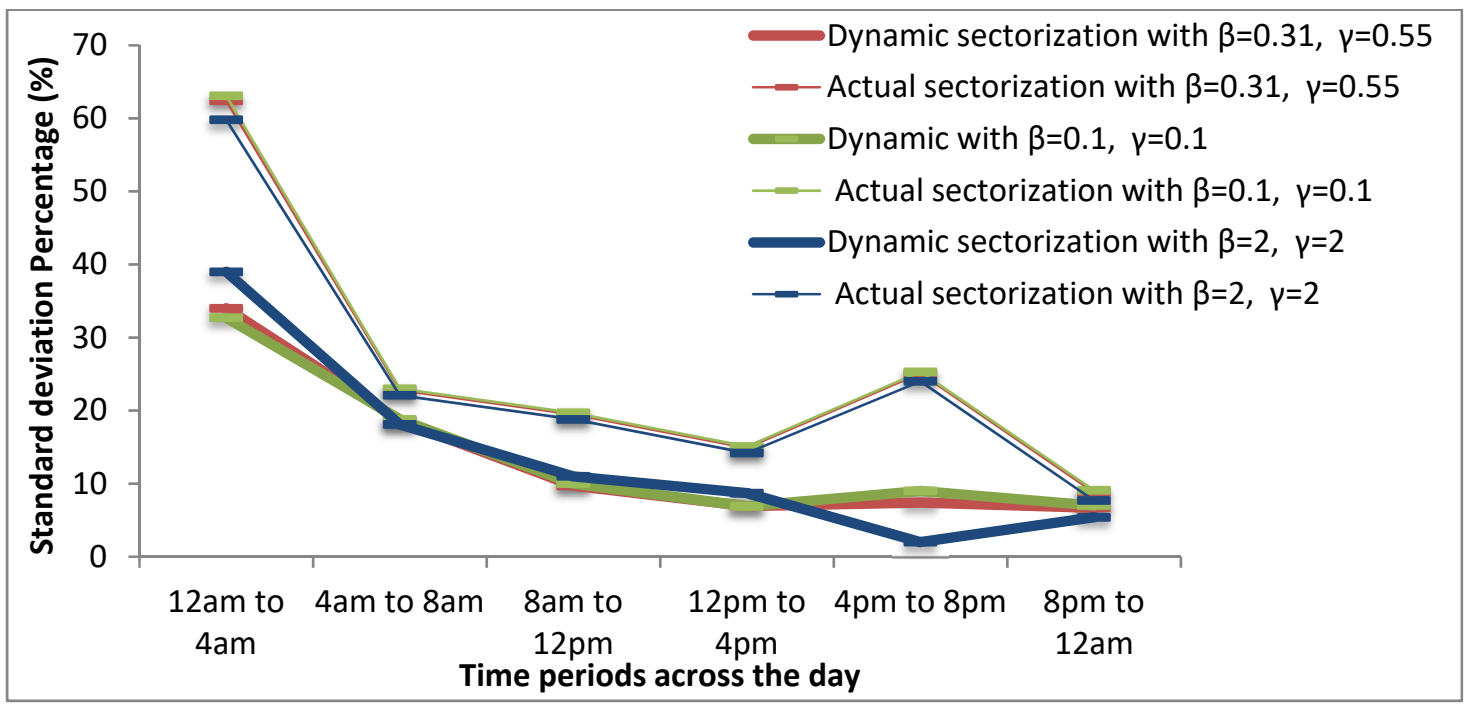

Table 1: Average values of $\boldsymbol{c}_{\boldsymbol{b a l}}$ and $\boldsymbol{\sigma}$

\begin{tabular}{|l|c|c|c|c|c|c|}
\hline & $\begin{array}{c}\text { Dynamic } \\
\text { sectorization } \\
\text { with } \boldsymbol{\beta}=\mathbf{0 . 3 1} \\
\text { and } \boldsymbol{\gamma}=\mathbf{0 . 5 5}\end{array}$ & $\begin{array}{c}\text { Dynamic } \\
\text { sectorization } \\
\text { with } \boldsymbol{\beta}=\mathbf{0 . 1} \\
\text { and } \boldsymbol{\gamma}=\mathbf{0 . 1}\end{array}$ & $\begin{array}{c}\text { Dynamic } \\
\text { sectorization } \\
\text { with } \boldsymbol{\beta}=\mathbf{2} \\
\text { and } \boldsymbol{\gamma}=\mathbf{2}\end{array}$ & $\begin{array}{c}\text { Actual } \\
\text { sectorization } \\
\text { with } \boldsymbol{\beta}=\mathbf{0 . 3 1} \\
\text { and } \boldsymbol{\gamma}=\mathbf{0 . 5 5}\end{array}$ & $\begin{array}{c}\text { Actual } \\
\text { sectorization } \\
\text { with } \boldsymbol{\beta}=\mathbf{0 . 1} \\
\text { and } \boldsymbol{\gamma}=\mathbf{0 . 1}\end{array}$ & $\begin{array}{c}\text { Actual } \\
\text { sectorization } \\
\text { with } \boldsymbol{\beta}=\mathbf{2} \\
\text { and } \boldsymbol{\gamma}=\mathbf{2}\end{array}$ \\
\hline $\begin{array}{l}\text { Average } \\
\boldsymbol{c}_{\boldsymbol{b a l}} \mathbf{( \% )}\end{array}$ & 22.4 & 23.1 & 22.7 & 35.7 & 36.6 & 34.5 \\
\hline $\begin{array}{l}\text { Average } \\
\boldsymbol{\sigma} \mathbf{( \% )}\end{array}$ & 13.9 & 14.1 & 14.0 & 25.6 & 25.9 & 24.4 \\
\hline
\end{tabular}


From Figs. 5 and 6 and Table 1, we note that $c_{b a l}$ and $\sigma$ are constantly lower for dynamic sectorization than that for the actual sectorization with the 3 groups of values of coefficients. Average $c_{b a l}$ is improved by $13.3 \%$ and $\sigma$ is improved by 8 flights for the results with the coefficients of $\beta=0.31$ and $\gamma=0.55 . c_{b a l}$ is improved by $13.5 \%$ and $\sigma$ is improved by 3 flights for the results with the coefficients of $\beta=0.1$ and $\gamma=0.1 . c_{b a l}$ is improved by $11.8 \%$ and $\sigma$ is improved by 30 flights for the results with the coefficients of $\beta=2$ and $\gamma=2$. Comparing $c_{b a l}$ and $\sigma$ for the actual sectorization with the various coefficients, we see that average $c_{b a l}$ is the highest at $36.6 \%$ when $\beta=0.1$ and $\gamma=0.1,35.7 \%$ when $\beta=0.31$ and $\gamma=0.55$ and the lowest at $34.5 \%$ when $\beta=2$ and $\gamma=2$. Based on the results for the actual sectorization, it seemed that the higher the coefficients, the more efficient the sectorization would be. However, when we take into account the average $\sigma$, we see that when $\beta=2$ and $\gamma=2, \sigma$ is significantly higher at 60 flights, compared to 15 flights and 6 flights when $\beta=$ 0.31 and $\gamma=0.55$ and $\beta=0.1$ and $\gamma=0.1$ respectively. When comparing the $c_{b a l}$ and $\sigma$ for dynamic sectorization with the various coefficients, we see that average $c_{b a l}$ is the lowest at $22.4 \%$ when $\beta=0.31$ and $\gamma=0.55,22.7 \%$ when $\beta=2$ and $\gamma=2$ and the highest at $23.1 \%$ when $\beta=0.1$ and $\gamma=0.1$ (Ong, 2016). Hence, the results show the importance of determining the optimal coefficients of coordination and conflict resolution with the assumed value of the coefficient of monitoring. Using the optimal coefficients would result in a more balanced workload among the sectors for the dynamic sectorization. The dynamic sectorization is better than actual sectorization for balancing the workload among sectors throughout the day, with an improvement of an average of $12.9 \%$ in the imbalance of workload among the sectors and $50 \%$ in the standard deviation.

As discussed in section 1, dynamic sectorization has not been implemented in Southeast Asia. Besides dynamic sectorization, other methods such as direct route airspace in this region can be also researched. In the direct route environment, there was a reduction in the ATCO's workload, because of fewer conflicts and lesser time spent in the sector (Aneeka and Zhong, 2018). Researchers in universities can be solution providers but are usually not decision makers for air transport systems. The implementation of these advanced approaches needs the support and approval from the decision makers. The research on air transport management in this region is still not enough. One challenge in this region might be the difficulty in finding collaborators in the aviation industry. However, the stakeholders can be expected to be even more supportive to researches, after more solutions to real-world problems are published (Zhong, 2018). 


\section{SUMMARY}

We studied flight trajectory optimization. Fuel savings were computed for selected routes and were compared with that of existing operations and flights simulations. The results obtained from the trajectories solutions provided by Model 1 resulted in a range of $300-1020 \mathrm{~kg}$ of fuel reduced per flight. For dynamic sectorization of Hanoi FIR, using the optimal coefficients resulted in the lowest imbalance of workload among the sectors, proving the importance of determining the optimal coefficients. Dynamic sectorization of airspace is consistently more advantageous than actual and static sectorization in terms of improving imbalance of workload among sectors and standard deviation.

\section{REFERENCES}

- Airbus (2005). A320 - Aircraft Characteristics Airport and Maintenance Planning. Airbus, Blagnac Cedex, France.

- Amin, R., Tang, J., Ellejmi, M., Kirby, S. and Abbass, H.A. (2013), "An evolutionary goalprogramming approach towards scenario design for air-traffic human-performance experiments", 2013 IEEE Symposium on Computational Intelligence in Vehicles and Transportation Systems (CIVTS), pp.64-71.

- Aneeka, S. and Zhong, Z.W. (2018). Modeling and Simulating Direct Route Environment in Singapore Airspace to Examine Conflicts, Aircraft Performance Output and Workload. Elixir Mechanical Engineering, 122, pp.51771-51773.

- Cavcar, M. (2000). The International Standard Atmosphere (ISA). [Online] Available at: http://home.anadolu.edu.tr/ mcavcar/common/ISAweb.pdf [Accessed 1 Decemeber 2015].

- FlightStats (2016). Global Flight Tracker, Status Tracking and Airport Information. [Online] Available at: http://www.flightstats.com/. [Accessed 15 February 2016].

- Foo, H.W.G. and Zhong, Z.-W. (2017). Analysis of the voice communication capacity for a free-route sectorless airspace. 2017 IEEE/AIAA 36th Digital Avionics Systems Conference, St Petersburg, FL, SEP 17-21.

- Foo, H.W.G. and Zhong, Z.-W. (2018), "A Transitional System for Operating Both Sectorless and Sectored Airspace in Southeast Asia", 18th Integrated Communications, Navigation and Surveillance Systems (ICNS) Conference, Herndon, VA, Apr 10-12, pp.3F11 - 3F1-11.

- International Civil Aviation Organization (2014). ICAO Carbon Emissions Calculator Methodology. [Online] Available at: http://www.icao.int/environmentalprotection/CarbonOffset/Documents/Methodology\%20ICAO\%20Carbon\%20Calculator v 7-2014.pdf [Accessed 1 April 2016].

- Kopardekar, P., Bilimoria, K. and Sridhar, B. (2007), "Initial concepts for dynamic airspace configuration", 7th AIAA aviation technology, integration and operations conference (ATIO), Belfast, Northern Ireland, pp.18-20.

- Li, J., Wang, T., Savai, M. and Hwang, I. (2009), "A spectral clustering based algorithm for dynamic airspace configuration", Proceedings of the 9th AIAA Aviation Technology, Integration and Operations (ATIO) Forum. American Institute of Aeronautics and Astronautics, pp.2009-7056. 
- Li, J., Wang, T., Savai, M. and Hwang, I. (2010). Graph-based algorithm for dynamic airspace configuration. Journal of guidance, control, and dynamics, 33, pp.1082-1094.

- Lim, W.-X. and Zhong, Z.-W. (2018). Re-Planning of Flight Routes Avoiding Convective Weather and the "Three Areas". IEEE Transactions on Intelligent Transportation Systems, 19, pp.868-877.

- Majumdar, A. and Ochieng, W. (2002). Factors affecting air traffic controller workload: Multivariate analysis based on simulation modeling of controller workload. Transportation Research Record: Journal of the Transportation Research Board, 1788, pp.58-69.

- $\quad \mathrm{Ng}$, H.K., Sridhar, B. and Grabbe, S. (2012), "A practical approach for optimizing aircraft trajectories in winds", 2012 IEEE/AIAA 31st Digital Avionics Systems Conference (DASC), pp.3D6-1-3D6-14.

- Ng, H.K., Sridhar, B., Grabbe, S. and Chen, N. (2011), "Cross-polar aircraft trajectory optimization and the potential climate impact", 2011 IEEE/AIAA 30th Digital Avionics Systems Conference (DASC), pp.3D4-1-3D4-15.

- Nita, M. and Scholz, D. (2012). Estimating the Oswald factor from basic aircraft geometrical parameters. presented at the Deutscher Luft- und Raumfahrtkongress, Estrel, Berlin.

- Ong, W.C.W. (2016). A Study of Air Traffic Management. Report of Nanyang Technological University, Singapore.

- Phyoe, S.M., Nguyen, N.Y., Aneeka, S. and Zhong, Z.W. (2016). The Impact of Population Growth on the Future Air Traffic Demand in Singapore. Proceedings of the International Conference on Computer Networks and Communication Technology, ACSR-Advances in Computer Science Research Vol. 54. P. A. Kowalski, S. Lukasik and P. Kulczycki. 54, pp.688-694.

- Raheja, D. and Zhong, Z.W. (2018). The causal relationship between GDP and air passenger traffic: Evidence from Singapore. International Journal of Transport Economics, 45, pp.83-95.

- Roth, B.A. and Mavris, D.N. (2001), "A work availability perspective of turbofan engine performance", presented at 39th AIAA Aerospace Sciences Meeting and Exhibition, Reno, NV.

- Savai, J.W., Wei, J., Li, J., Wang, T. and Hwang, I. (2010), "Multilevel graph partitioning algorithm for dynamic airspace configuration", the Proceedings of the AIAA Aviation Technology, Information, and Operations Conference, Fort Worth, TX.

- Sheth, K., Amis, T., Gutierrez-Nolasco, S., Sridhar, B. and Mulfinger, D. (2013). Development of a probabilistic convective weather forecast threshold parameter for flightrouting decisions. Weather and Forecasting, 28, pp.1175-1187.

- Sun, W.Y. (2016). A modified flight optimization methodology for ASEAN application. Report of Nanyang Technological University, Singapore.

- Tee, Y.Y. and Zhong, Z.W. (2018). Modelling and simulation studies of the runway capacity of Changi Airport. Aeronautical Journal, 122 pp.1022-1037.

- Thanh Nien News (2016). Vietnam rushes for new north-south air path as traffic grows. [Online] Available at: http://www.thanhniennews.com/travel/vietnam-rushes-for-newnorthsouth-air-path-as-traffic-grows-58368.htm. [Accessed 03 March 2016].

- Trong, N.V., Aneeka, S. and Zhong, Z.W. (2016). Relationship of Sector Size and Sector Partitioning to Controller Workload. The 18th International Conference on BESSH-2016, ECBA-2016 \& MMHS-2016, Singapore.

- Turgut, E.T., Karakoc, T.H. and Hepbasli, A. (2009). Exergoeconomic analysis of an aircraft turbofan engine. International Journal of Exergy, 6, pp.277-294.

- Vietnam Aeronautical Information Centre (2015). AERONAUTICAL INFORMATION PUBLICATION (AIP). [Online] Available at: http://vnaic.vn/images/stories/vnaic.vn/SanPhamDichVu/AIPVietNam/Related\%20Links/ 2015-03-AMDT/html/eAIP/ENR-2.1-en-GB.html [Accessed 15 February 2016]. 
- Von Luxburg, U. (2007). A tutorial on spectral clustering. Statistics and computing, 17, pp.395-416.

- Wang, T., Li, J. and Hwang, I. (2010). A Sectorization Method for Dynamic Airspace Configuration. presented at the AIAA Guidance, Navigation, and Control Conference, Toronto, Canada.

- Xie, Z. and Zhong, Z.W. (2016a). Aircraft Path Planning under Adverse Weather Conditions. 2016 3rd International Conference on Mechanics and Mechatronics Research, MATEC Web of Conferences Vol. 77 Article Number: UNSP 15001. D. Zhang, B. Zi, G. Cui and H. Ding. 77.

- Xie, Z. and Zhong, Z.W. (2016b). Simulated Civil Airplane Visual Conspicuity Experiments During Approaching and Departure in the Airport Vicinity. 2016 7th International Conference on Intelligent Systems, Modelling and Simulation. D. Aldabass, T. Achalakul, R. Sarochawikasit and S. PromOn, pp.279-282.

- Zhong, Z.W. (2018). Overview of recent developments in modelling and simulations for analyses of airspace structures and traffic flows. Advances in Mechanical Engineering, 10, Article Number: 1687814017753911.

- Zhong, Z.W., Salleh, S.R., Chow, W.X. and Ong, Z.M. (2016). Studies of air traffic forecasts, airspace load and the effect of ADS-B via satellites on flight times. Conference on Advanced Free-Space Optical Communication Techniques and Applications II, Edinburgh, Scotland, Proceedings of SPIE, Volume: 9991, Article Number: UNSP 99910B.

- Zhong, Z.W., Varun, D. and Lin, Y.J. (2017). Studies for air traffic management R\&D in the ASEAN-region context. Journal of Air Transport Management, 64, pp.15-20. 